\title{
Título: La corrupción de la administración de Justicia destruye la satisfacción con el funcionamiento de la democracia.
}

\begin{tabular}{|c|c|}
\hline ORCID & $\begin{array}{c}\text { Manuel Núñez } \\
\text { Investigador en formación }\end{array}$ \\
& $\begin{array}{c}\text { Escuela Internacional de Doctorado de la Uned } \\
\text { Programa de Doctorado Análisis de Problemas Sociales } \\
\text { mnunez266@alumno.uned.es }\end{array}$ \\
\hline
\end{tabular}

\section{Resumen}

La satisfacción con el funcionamiento de la Democracia es un indicador relevante de la salud de la Democracia. Esa satisfacción seguramente es multidimensional y depende de cómo es percibido el rendimiento de las diferentes instituciones. Entre las instituciones que aportan una contribución relevante a esa satisfacción, sin duda, está la Administración de Justicia. Distorsiones en su funcionamiento afectan a la satisfacción con la democracia. En este artículo mostramos como la percepción de la corrupción de la Administración de Justicia, afecta negativamente a la satisfacción con el funcionamiento de la Democracia directamente e indirectamente a través de la percepción de la igualdad ante la ley y el derecho a la Justicia.

\begin{abstract}
Satisfaction with Democracy functioning is a relevant indicator of Democracy's health. This satisfaction is surely multidimensional and depends on how the performance of the different institutions is perceived. Among the institutions that make a significant contribution to that Satisfaction is, undoubtedly, the Justice Administration. Distortions in its functioning, like corruption, affect negatively this contribution. In this article we show how the perception of corruption in the Administration of Justice negatively affects satisfaction with the functioning of Democracy directly; and indirectly, through the perception of equality before the law and the right to Justice.
\end{abstract}

\section{Introducción}

En nuestro artículo "Corrupción: ampliando el alcance" (Nuñez, 2021) hemos propuesto una visión de la corrupción que supera los estrechos límites en los cuales la visión "mainstream" la ha mantenido, para recuperar su potencial explicativo en el mantenimiento y cambio de régimen. En "La Corrupción como estrategia de la Antipolítica" (Nuñez, 2022) hemos mostrado como la saliencia de la corrupción afecta a dos pilares de la democracia, el interés por la política y la legitimidad de la competencia multipartidaria. Como continuación de este último estudio vamos a analizar ahora como la prevalencia de la corrupción afecta a la satisfacción con la democracia.

Evidentemente la satisfacción con la democracia es una variable relevante en la continuidad del régimen democrático, toda vez que si cualquier régimen -incluidos los autoritarios- requieren cierta aquiescencia -aunque sea "pasiva"- de los "dominados" para su supervivencia, la 
democracia, lo requiere especialmente. Si el hombre político está más satisfecho, de acuerdo con la teoría de la elección racional, en menos probable que esté tentado por la rebelión.

Diferentes fuentes muestran que la satisfacción con la democracia está cayendo en el mundo. Cada cual señala como causas de esta caída de la satisfacción las que mejor encajan en su visión del mundo. Pero entre ellas no ocupa un lugar menor la corrupción sistémica (una vez más recordamos que es necesario no confundirla con la corrupción generalizada) que dificulta o hace imposible al "soberano durmiente" ejercer su soberanía para cambiar la dirección de la ruptura de las condiciones en las cuales la voluntad general puede expresarse, a saber, la existencia de igualdad de palabra y de sufragio activo y pasivo. Y dentro de esa corrupción sistémica, ocupa un lugar significativo la corrupción de la administración de justicia. Por razones que en seguida se harán evidentes, la corrupción de la Administración de Justicia, afectará especialmente a la satisfacción con la democracia. Pero comprender este nexo, requiere entender cuál es el nexo entre la Administración de Justicia y el Poder Político.

Una de las retóricas que más tracción ha ganado en los últimos años, especialmente alimentada por una fracción no menor de los funcionarios en plantilla de la Administración de Justicia, es la substracción de esta de la égida del Soberano, que, en una Democracia, es el Pueblo constituido en Asamblea Soberana -ni multitud ni gente-. En una interpretación perversa, e histórica y constitucionalmente incorrecta, del principio de separación (que no división) de poderes, se pretende que los funcionarios en la plantilla de la Administración de Justicia, encarnan un poder soberano independiente.

Toda vez que no es posible encontrar cual es el origen de este poder soberano, distinto del Pueblo, tal y como establece el Artículo 1. Apartado 2. "La soberanía nacional reside en el pueblo español, del que emanan los poderes del Estado", de la Constitución del 78, se apalanca sobre dos "disparates" recogidos en la Constitución Española de 1978. El primero de ellos aparece en el Artículo 117. 1. de la misma Constitución se dice "La justicia emana del pueblo y se administra en nombre del Rey por Jueces y Magistrados integrantes del poder judicial", de donde pretende deducirse alguna relación especial, olvidando por lo demás, que en otro lugar de la Constitución se dice que todos los actos del Rey deberán ser refrendados por algún miembro del Gobierno. Por tanto, recabar esa relación especial, requeriría refrendo del Gobierno, lo cual es una contradicción en toda regla. Pero aún más insidioso es el desplazamiento, de lo que dice el Artículo 122 de la misma Constitución. Si bien este artículo establece que de los 21 miembros del Consejo General del poder judicial, 12 deberán ser funcionarios en activo en la plantilla de la administración de Justicia (jueces o magistrados), nada dice sobre el modo como se eligen. Se dice que los nombra el Rey (cuyos actos necesitan ser refrendados por un miembro del Gobierno). Por tanto, deducir que deben ser elegidos por los propios funcionarios (jueces y magistrados) en la plantilla de la administración de Justicia, carece de fundamento alguno. Aún así, al no haberse previsto en la misma Constitución, ningún procedimiento especial, por ejemplo, una "Institución Plebeya", para el Juicio de conductas delictivas entre los funcionarios (jueces y magistrados), estos siguen gozando de un privilegio, del que el resto de los funcionarios, con buen criterio, han sido privados, los "Tribunales de Honor". Evidentemente este nombre no aparece por ningún lugar, toda vez que, esos funcionarios (jueces y magistrados) son aparentemente sometidos a "procedimientos ordinarios". Los discursos que obliteran el texto constitucional y a la ausencia de instituciones diseñadas para prevenir y perseguir la corrupción judicial seguramente tienen que ver con la baja prevalencia de las condenas sobre este cuerpo de funcionarios en los más de 40 años de vigencia de la Constitución, así como la dudosa solvencia de algunas de las que han sido emitidas. Esto crea las condiciones para la 
propagación de diferentes tipos de corrupción en la administración de justicia. Los "demonios" están en todas partes donde se ejerce poder político y los funcionarios de la administración de justicia no son una excepción.

Por otra parte, que el poder ejercido por los funcionarios en la plantilla de la administración de Justica es político, y como tal, es un ejercicio de la violencia política, simbólica y material, contra los que los discursos auto-justificativos que reclaman la naturaleza "abstracta", "universal", "angelical" y por tanto "incontaminada" del juicio judicial, quedará de manifiesto a lo largo de los párrafos que siguen.

Como los ciudadanos establecen esas mediaciones, especialmente como la corrupción de la administración de justicia ataca un principio constitucional esencial -la igualdad ante la ley- será parcialmente desentrañado en la parte empírica de este artículo.

\section{Marco Teórico}

Una de las formulaciones más brillantes de cuál es el problema fundamental que la política -y la política democrática no es una excepción-, nos la proporciona (Kant, 2011) en "La paz perpetua" con su famosa reflexión sobre un "pueblo de demonios" ${ }^{1}$. En esta definición aparecen los elementos esenciales que podemos encontrar a lo largo de muchos autores de los que han escrito sobre la teoría política:

- Pueblo de demonios (los demonios por definición tienen entendimiento), es decir, en especial relación con el mal.

- Seres racionales que para su supervivencia requieren leyes generales: capaces de calcular lo que les conviene.

- Tendencia de cada uno de estos seres racionales a eludir, en secreto, las leyes en interés propio: vicios privados.

- Tendencias privadas opuestas que se contienen con una Constitución: contrato.

- Para que en su comportamiento público el éxito sea el mismo que si no tuvieran esas malas tendencias: vicios privados que se transforman en virtudes públicas.

- No se trata de la mejora moral del ser humano, sino del mecanismo de la naturaleza: lógica de relaciones, no de substantivas identidades.

- Cómo se puede utilizar dicho mecanismo en el hombre para orientar la oposición de las tendencias no pacíficas de un pueblo: tendencia intrínseca a la violencia, es decir, a imponerse unos a los otros.

- Se obliguen a sí mismos a someterse a leyes coactivas, debiendo generar así la situación de paz en la que las leyes tienen fuerza: obediencia voluntaria.

Pero la "obediencia voluntaria" que parece "natural" -y ha generado confusión a mentes tan sagaces como (Boétie, 1995)- oculta la presencia constante, implícita o explicita, de la violencia. Simplificando mucho, hay cuatro medios para conseguir "cooperación" (en el continuo "voluntaria - forzada"): seducción, llamada de atención ("warning"), amenaza y fuerza física. Los tres primeros forman parte de la retórica: la formulación se asocia a priori a un bien deseado - rechazado (en el continuo obtener-perder, valor positivo-negativo) pero se mantiene la opción de elegir. El cuarto deja de ser retórico porque desaparece la posibilidad de elegir. En términos de Greimas (Greimas, 1987) (Greimas \& Fontanille, 2002), la dimensión legítima de la autoridad, es la inclusión de un marco narrativo (retórico) en un mismo hecho físico-el ejercicio de la violencia-. 
Evidentemente el ejercicio legitimo de la autoridad implica poder político. Y el poder político no es una "mercancía" como las demás ${ }^{2}$ aunque ciertas definiciones generales como la de (Weber, 2002) -tan citada $^{3}$, - pueda inducir a pensar lo contrario, especialmente si endosa con independencia de su definición de dominación ${ }^{4}$. Y es que acostumbrados a asistir al hecho de que una orden es seguida por una ejecución, tendemos a pensar que la obediencia está causada por la orden", cuando es todo lo contrario. (Tarde, 2003) lo expresa así : "Le Pouvoir n'est en somme que le privilège de ce faire obéir»" (pag. 59). Por tanto "Le pouvoir politique réside dans l'opinion des gouvernés qu'il faut persuader et satisfaire à la fois " (pág. 106). Y por último, "Une société, comme une personne, ne dispose, a un moment donne, que d'une certaine quantité d'obéissance et de confiance, qui se répartir entre les divers pouvoirs auxquels on se soumet " (pág. 83). Este enfoque permite mirar desde otra perspectiva los conceptos de "problema" e "interés". Los "problemas" serán verdaderos o falsos de acuerdo con una determinada definición operativa; por ejemplo, existe o no existe corrupción; y los problemas pueden existir -existen- con independencia de su reconocimiento-. Otro asunto diferente es el interés, en el sentido de "s'intéresser", es decir, hacer del problema (que por supuesto puede ser un problema falso) un motivo de obediencia o desobediencia a la orden y/o reclamar el cambio de orden y/o cambiar/eliminar a quién dicta la orden (que pasa a ser definido como parásito). En este sentido, todo interés es una creencia ${ }^{7}$, que no es verdadera o falsa, sino relativa, y está sometido a las leyes (o quizás a la ausencia de ellas, porque es resultado de la innovación ${ }^{8}$ ) de acuerdo con las que se produce. En este sentido, todo líder de un movimiento social o político es un innovador, a igual título que en cualquier otro dominio, aunque su rasgo distintivo es "crear coaliciones y ponerlas en movimiento". Y esta actividad, como se ha señalado en términos más generales, para la creación de conocimiento (Sloman \& Fernbach, 2017), no es nunca una actividad individual, sino colectiva ${ }^{9}$. Y por supuesto en esa tarea la libertad irrestricta no siempre tiene cabida pues, como dice (Tarde, 2003) «on n'est pas toujours maître de croire de qu'on désirait croire, ce qu'on aurait intérêt à croire» (pág. 193) ${ }^{10}$. "La Politique ${ }^{11}$, en somme, est cette partie de l'activité d'un peuple ou d'una fraction de peuple qui a pour objet propre e direct /.../...) la domination sur d'autres ou tout au moins l'affranchissement, la domination sur d'autres peuples ou d'autres fractions du même peuple, ou l'affranchissement d'une servitude imposée par d'autres peuples ou d'autres fractions du même peuple» (pág 210).

Por eso, cuando hablamos de fuerza, no solamente hablamos de fuerza física directa, sino también la que se oculta bajo la forma de derecho constituido y derecho constituyente ${ }^{12}$. Todo el edificio del Derecho está siempre en esta dualidad; donde más claro se percibe es en la Corte Penal, donde se introducen marcos narrativos del teatro, para crear la apariencia retórica (y ocultar la decisional) (Lagasnerie, 2016). Recuérdese, que como hemos recordado con la ayuda de Greimas, cuando desaparece la apariencia de elección, desaparece el campo de la retórica y solo queda el de la violencia. Aunque el Estado Moderno (democrático) ha tendido a ocultar e incluso demonizar, la violencia como medio de resolver conflictos, la violencia está presente en cada decisión del Estado constituido, incluso (o sobre todo) cuando se manifiesta como administración de justicia. (Lagasnerie, 2016) lo describe con precisión:

"Penser la justice, c'est penser une " pratique » dont l'" objectif » est de faire souffrir. Si l'on veut nommer ce qui se passe dans un tribunal, il faut dire : exercer de la violence (.../...) La Justice est le lieu d'une agresión».

A la vista de la relación entre la "justicia", la violencia y el sufrimiento, no resultará especialmente raro que la percepción de que la justicia es corrupta, afecte a la satisfacción con 
la democracia. Y tampoco resulta estrambótico, ni una ocurrencia, decir que la "igualdad ante la ley" es la igualdad ante el sufrimiento.

\section{El presente análisis}

En este contexto teórico, en este documento vamos a testar una nueva hipótesis -que correlativamente vamos a nombrar como Hipótesis 3- sobre la corrupción en España. Vamos a utilizar para ellos datos del estudio "Ética pública y corrupción" (Estudio n. 2826) elaborado por el Centro de Investigaciones Sociológicas (CIS, 2009) que ya utilizamos, para testar las Hipótesis 1 y 2 en (Nuñez, Corrupción como estrategia de la anti-política, 2022).

\section{Hipótesis 3:}

La percepción de la corrupción del sistema judicial afecta directamente a la satisfacción con el funcionamiento de la democracia; indirectamente afecta a la satisfacción con el funcionamiento de la democracia a través de su impacto en la percepción de que los españoles son iguales ante la ley, la justicia castiga a todos por igual; y la justicia tarda, pero llega.

\section{Procedimiento}

Para testar las Hipótesis 3 vamos a construir un modelo "causal" con variables manifiestas. Los modelos "causales" con variables manifiestas, conocidos por varios nombres en la literatura, tienen una larga tradición en las ciencias sociales (Blalock, 1985) (Jaccard \& Jacoby, 2020). En los últimos años, han recibido un nuevo impulso, con enfoques diversos (Pearl, 2000) (Cartwright, 2007) (Morgan \& Winship, 2015) (Pearl, Glymour, \& Jewell, 2016) (Pearl2016) (Pearl \& Mackenzie, 2018) (Hernan \& Robins, 2023) (Hernan2023). De todos estos enfoques, en nuestro caso, vamos a seguir el que propone (Hayes, 2022, pág. 10) como "conditional process analysis": "Conditional process analysis is used when one's research goal is to describe the conditional nature of the mechanism or mechanisms by which a variable transmits its effect on another and testing hypotheses about such contingent effects". Hay dos tipos de análisis condicional, mediación y moderación, que pueden ser realizados conjunta o separadamente. En el particular problema que nos ocupa, vamos a ajustar un modelo de mediación para determinar el efecto directo e indirecto de la percepción de la corrupción de la administración de Justicia sobre la satisfacción con el funcionamiento de la Democracia.

Del estudio "Ética pública y corrupción" (Estudio n.․ 2826) elaborado por el Centro de Investigaciones Sociológicas (CIS, 2009) tomaremos las siguientes variables:

\begin{tabular}{|c|c|}
\hline Independientes & Dependientes \\
\hline $\begin{array}{l}\text { (P22) Percepción de la generalización de la corrupción de la } \\
\text { Administración de Justicia. }\end{array}$ & \multirow{4}{*}{$\begin{array}{l}\text { (P5) Satisfacción con el } \\
\text { funcionamiento de la } \\
\text { democracia en España. }\end{array}$} \\
\hline (P19) Los españoles son iguales ante la ley. & \\
\hline $\begin{array}{l}\text { (P1801) El sistema judicial castiga a los culpables sin importar } \\
\text { quienes son. }\end{array}$ & \\
\hline (P1802) La justicia tarda, pero llega & \\
\hline
\end{tabular}

Tabla 1: Variables que entran en el contraste de la Hipótesis 3. 
Las frecuencias respectivas de cada variable aparecen en la Tabla 2. La pregunta P22 ha sido recodificada a tres categorías: 1 (Muy extendida + Bastante extendida), 2 (Algo extendida), 3(Poco extendida + Nada extendida + No hay corrupción).

\section{P5 Satisfacción con el funcionamiento de la Democracia en España \%}

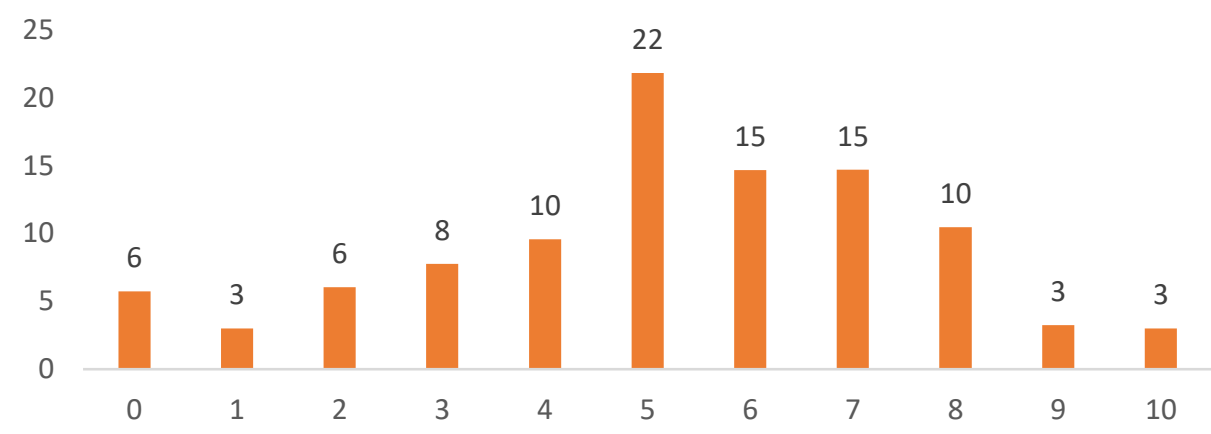

P22_r Corrupción en la Administración de Justicia \%

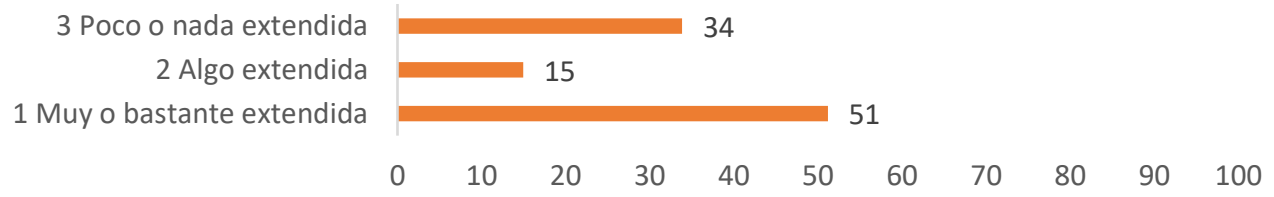

P1904 Los españoles son iguales ante la ley \%

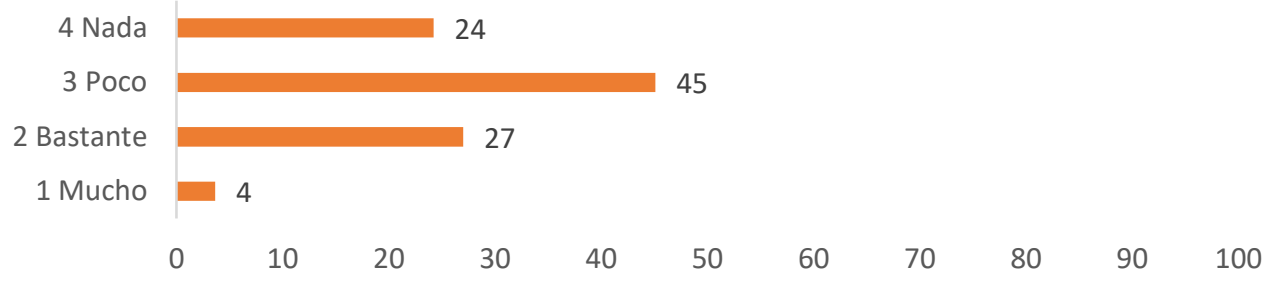

P1801 El sistema judicial castiga a los culpables sin importar quienes son \%

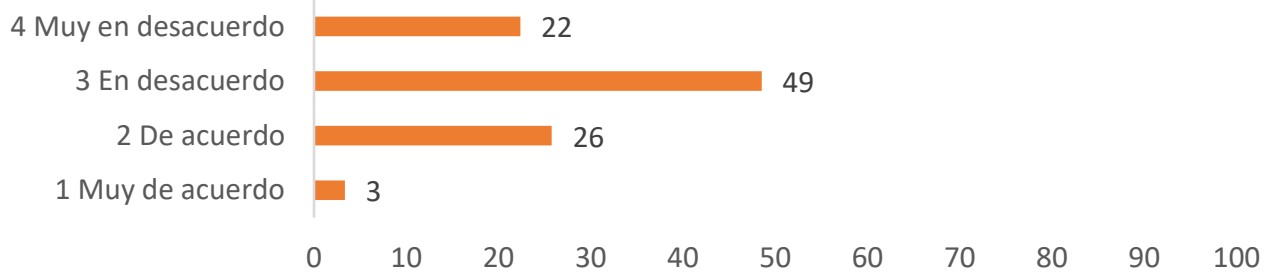




\section{P1802 La Justicia tarde pero llega \%}

4 Muy en desacuerdo

3 En desacuerdo

2 De acuerdo

1 Muy de acuerdo

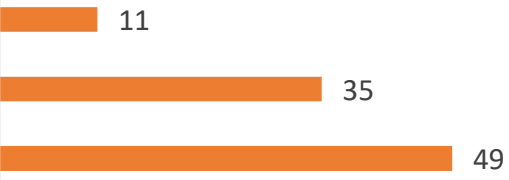

49

$\begin{array}{lllllllllll}0 & 10 & 20 & 30 & 40 & 50 & 60 & 70 & 80 & 90 & 100\end{array}$

Gráfico 1: Frequencias de las variables

En la Tabla 3 aparece la matriz de correlaciones entre las variables, que nos indica que:

- A menor percepción de corrupción en la administración de justicia mayor satisfacción con la democracia.

- Cuanto menor es la percepción de que los españoles son iguales ante la ley menor satisfacción con la democracia.

- E igualmente cuanto menor es la percepción de que la justicia es igual para todos y que la justicia llega, aunque sea tarde, menor satisfacción con la democracia.

Matriz de correlaciones con las variables recodificadas

\begin{tabular}{c|ccccc}
\hline Variables & P5 & P2202_r & P1904_r & P1801_r & P1802_r \\
\hline P5 & 1.000 & Polyserial & Polyserial & Polyserial & Polyserial \\
P2202_r & 0.279 & 1.000 & Polyserial & Polyserial & Polyserial \\
P1904_r & -0.223 & -0.327 & 1.000 & Polychoric & Polychoric \\
P1801_r & -0.188 & -0.290 & 0.582 & 1.000 & Polychoric \\
P1802_r & -0.179 & -0.305 & 0.462 & 0.616 & 1.000 \\
\hline
\end{tabular}

Tabla 2: Matriz de correlaciones entre las variables que entran en el modelo

En el modelo de mediación, la percepción de la corrupción del sistema judicial (P2202_r (x)) actúa como variable independiente y la satisfacción con la democracia (P5 (y)), como variable dependiente. Las otras tres variables, la percepción de que los españoles son iguales ante la ley (P1904_r (m1)), la justicia castiga a todos por igual (P1801_r (m2) y la justicia tarda, pero llega (P1802_r (m2)), actuarán como mediadores.

El modelo de mediación aparece representado como modelo conceptual en la Figura 1. 


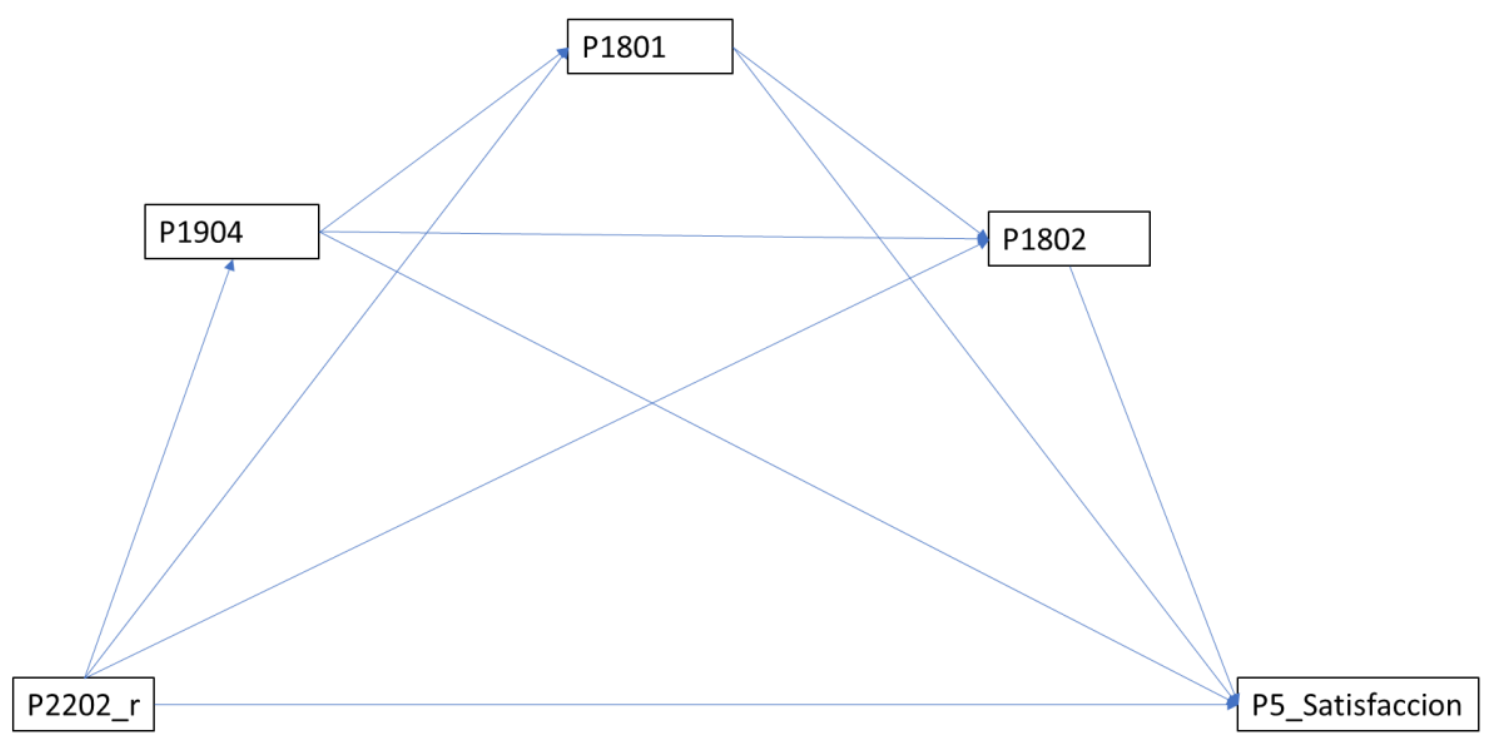

Figura 1: Gráfico del modelo conceptual de mediación con coeficientes (M6)

La variable P2202_r será tratada en el modelo como multi - categórica, toda vez que estamos interesados en mostrar como impacta el paso de los diferentes grados de percepción de corrupción en la administración de Justicia sobre la satisfacción con el funcionamiento de la Democracia. La codificación usada para determinar esos efectos es tipo indicador, donde la categoría base es la 1 (Muy + Bastante) tal y como aparece en la Tabla 3.

\begin{tabular}{rcc}
\hline La corrupción en la administración de Justicia & X1 & X2 \\
\hline 1 Muy o bastante extendida & 0 & 0 \\
2 Algo extendida & 1 & 0 \\
3 Poco o nada extendida & 0 & 1 \\
\hline
\end{tabular}

Tabla 3. Codificación de la variable P22_r

De la inspección de la representación gráfica del modelo y teniendo en cuenta el sistema de codificación de la P22_r, se deduce que necesitamos un total de 14 ecuaciones, cuyos coeficientes aparecen detallados en la Tabla 4.

\begin{tabular}{r|cccc}
\hline \multicolumn{5}{c}{ Coeficientes Parciales } \\
\hline P2202_r & P1904 & P1801 & P1802 & P5 \\
\hline X1 & & & & 0.7241 \\
X2 & & & & 0.9579 \\
X1 & -0.2050 & -0.0954 & -0.1089 & \\
X2 & -0.4282 & -0.1978 & -0.1456 & \\
P1904 & & 0.3609 & 0.1609 & -0.3471 \\
P1801 & & & 0.373 & -0.1208 \\
P1802 & & & & -0.2232 \\
\hline
\end{tabular}

Tabla 4. Coeficientes parciales del modelo del Gráfico 1. 
Todos los coeficientes son significativos a $\mathrm{P}=.005$ salvo $\mathrm{P} 1801-->\mathrm{P} 5(\mathrm{P}=0.1267)$. En el intervalo de confianza construido con bootstrapping todos los coeficientes aparecen como significativos, salvo este mismo coeficiente P1801-->P5, en cuyo intervalo de confianza si aparece el 0 (LLCl ,2759; ULCI ,0343). En aras de la brevedad, salvo en el caso de los coeficientes directos que aparecen en la Tabla 5, no mostraremos ni los valores de $\mathrm{P}$ ni los intervalos de confianza construidos con bootstrapping para el resto de los coeficientes.

En la Tabla 5 aparece el detalle para los efectos relativos directos (indicado en el Gráfico 1 por la flecha que va directamente de la P2202_r a la P5). El Ómnibus Test sobre los efectos directos, también arroja resultados significativos.

\begin{tabular}{llrrrrrrr}
\hline Efectos directos & & & & & & & & \\
\hline & & Coeff. & se & t & P & LLCl & ULCl & c_ps \\
& X1 & 0.7241 & 0.1595 & 4.5394 & 0 & 0.4113 & 1.037 & 0.2992 \\
X2 & 0.9579 & 0.1209 & 7.9231 & 0 & 0.7208 & 1.195 & 0.3958 \\
\hline
\end{tabular}

Ómnibus test del modelo de efectos directos

$\begin{array}{rrrrr}\text { R2-chng } & F & \text { df1 } & \text { df2 } & P \\ 0.032 & 33.719 & 2.000 & 1921.000 & 0.000\end{array}$

Tabla 5. Ajuste del modelo de efectos relativos directos

En la Tabla 6 aparece el detalle de todos los efectos relativos indirectos. Como puede apreciarse el intervalo de confianza nos dice que todos son significativos salvo los que incluyen la P1801 sin la P1802 (lo que sugiere que la percepción de que el sistema judicial castiga a todos por igual requiere para afectar a la percepción de la satisfacción con el funcionamiento de la democracia la idea de que la justicia llega tarde, pero llega).

\begin{tabular}{lrrrr}
\hline Efectos Relativos Indirectos & & & & \\
\hline P2202_r-->P1904-->P5 & & & LLCl & ULCl \\
& X1 & 0.0711 & 0.031 & 0.122 \\
& X2 & 0.1486 & 0.081 & 0.219 \\
P2202_r-->P1801-->P5 & & & LLCl & ULCl \\
& X1 & 0.0115 & -0.004 & 0.038 \\
& X2 & 0.0239 & -0.007 & 0.062 \\
P2202_r-->P1802-->P5 & & & LLCl & ULCl \\
& X1 & 0.0243 & 0.002 & 0.057 \\
& X2 & 0.0325 & 0.007 & 0.065 \\
P2202_r-->P1904-->P1801-->P55 & & & LLCl & ULCl \\
& X1 0.0089 & -0.0024 & 0.024 \\
& X2 & 0.0187 & -0.0054 & 0.0453 \\
P2202_r-->P1904-->P1802-->P5 & & LLCl & ULCl \\
& X1 0.0074 & 0.0014 & 0.0159 \\
& X2 & 0.0154 & 0.0033 & 0.0299 \\
P2202_r--P1801-->P1802-->P55 & & & LLCl & ULCl
\end{tabular}




\begin{tabular}{rlrr} 
X1 & 0.0079 & 0.0000 & 0.0202 \\
X2 & 0.0165 & 0.0035 & 0.0321 \\
P2202_r-->P1904-->P1801-->P1802-->P5 & LLCI & ULCI \\
X1 & 0.0062 & 0.0013 & 0.0126 \\
X2 & 0.0129 & 0.0029 & 0.0236 \\
\hline
\end{tabular}

Tabla 6. Efectos relativos indirectos

Cuando ponemos juntos los efectos directos y los indirectos, apreciamos, en la Tabla 7 que el modelo es significativo en todos los indicadores considerados. $Y$ apreciamos también que los coeficientes son más altos que cuando consideramos los efectos directos únicamente, indicación inequívoca de que la percepción en la corrupción de la administración de Justicia actúa tanto directa como indirectamente sobre la Satisfacción con la Democracia.

\begin{tabular}{rrrrrrrrr}
\hline \multicolumn{1}{l}{ ljuste del modelo de efectos totales } & & & & & & \\
\hline $\mathrm{R}$ & $\mathrm{R}-\mathrm{sq}$ & MSE & $\mathrm{F}$ & $\mathrm{df1}$ & $\mathrm{df2}$ & $\mathrm{P}$ & \\
0.237 & 0.056 & 5.536 & 56.990 & 2.000 & 1924.000 & 0.000 & \\
& & & & & & & \\
& coeff & se & $\mathrm{t}$ & $\mathrm{P}$ & $\mathrm{LLCl}$ & $\mathrm{ULCl}$ & $\mathrm{C} \_\mathrm{ps}$ \\
Constante & 4.623 & 0.075 & 61.659 & 0.000 & 4.482 & 4.777 & \\
$\mathrm{X} 1$ & 0.862 & 0.161 & 5.352 & 0.000 & 0.546 & 1.177 & 0.356 \\
$\mathrm{X} 2$ & 1.226 & 0.118 & 10.411 & 0.000 & 0.995 & 1.457 & 0.507 \\
\hline
\end{tabular}

Omnibus test del modelo de efectos totales

$\begin{array}{rrrrr}\text { R2-chng } & F & d f 1 & d f 2 & P \\ 0.056 & 56.990 & 2.000 & 1924.000 & 0.000\end{array}$

Tabla 7. Ajuste del Modelo de Efectos Totales

Por tanto, a la vista de todos los resultados obtenidos, podemos aceptar la Hipótesis 3.

La satisfacción con el funcionamiento de la Democracia sin duda depende de otros factores, aparte de los que hemos considerado aquí. Pero, tal y como hemos mostrado, la percepción de que la administración de Justicia está poco o nada afectada por la corrupción es un factor que contribuye positivamente a incrementar la satisfacción. La mayor parte del efecto es directo, pero también es importante el efecto indirecto construido a través del resto de las variables consideradas, especialmente a través del impacto sobre la percepción de igualdad ante la ley.

\section{Discusión}

Las evidencias aportadas aquí, refuerzan la relevancia de las ideas comunicadas en (Nuñez, Corrupción como estrategia de la anti-política, 2022). Y por supuesto, dado que apalancan sobre la misma fuente de datos, adolecen de las mismas debilidades ya señaladas, especialmente la naturaleza correlacional de los datos y la ausencia de información detallada sobre las propiedades psicométricas de las escalas utilizadas. Aún así, permiten documentar el impacto potencial de la percepción de la corrupción en los poderes del Estado que habitualmente no son el foco de la comunicación pública. En efecto, pese al potencial efecto deletéreo de la corrupción en la administración de Justicia, en el discurso público esta aparece poco o nada, dado que se suele primar el discurso sobre la corrupción del poder ejecutivo, porque ello sirve a los 
rendimientos electorales del opositor. Esto es particularmente grosero en países donde la corrupción judicial, aparte de su recorrido propio, camina hombro con hombro con la corrupción del poder ejecutivo.

Pero desde el punto de vista de la calidad de la democracia, la corrupción "silenciosa" de otras "ramas" del poder -muy especialmente del judicial-, que, como hemos visto -aunque sus integrantes y voceros no gusten de presentarse así-, se caracteriza por el ejercicio de la violencia, afecta negativamente a la satisfacción con el funcionamiento de la democracia, porque permite visualizar, justamente, el ejercicio arbitrario de la violencia. Y la democracia, en tanto que requiere el "imperio de la ley" se compadece mal con el ejercicio arbitrario de la violencia por funcionarios corruptos que, supuestamente, solo se deben al imperio de la ley, no a su corrupción.

\title{
Notas
}

\begin{abstract}
${ }^{1}$ La cita que nos interesa dice: "El problema de la Constitución del Estado tiene solución, incluso para un pueblo de demonios, por muy fuerte que suene (siempre que éstos tengan entendimiento), y se formula de la siguiente manera: "Organizar a un cierto número de seres racionales que para su supervivencia exigen finalmente leyes generales, donde sin embargo cada uno de ellos en secreto tiende a eludirlas, $e$ instituir su Constitución de manera que, a pesar de que sus tendencias privadas sean opuestas, éstas se contengan mutuamente para que en su comportamiento público el éxito sea el mismo que si no tuvieran esas malas tendencias». Debe de tener una solución este problema. Pues no se trata de la mejora moral del ser humano, sino sólo del mecanismo de la naturaleza; la tarea está en saber cómo se puede utilizar dicho mecanismo en el hombre para orientar la oposición de las tendencias no pacíficas de un pueblo de manera que se obliguen a sí mismos a someterse a leyes coactivas, debiendo generar así la situación de paz en la que las leyes tienen fuerza".
\end{abstract}

${ }^{2}$ De la "teoría liberal" surgen teorías del poder, ciertamente confusas, que generalizan el concepto de poder a toda clase de relación social, asimilando poder a "influencia, reputación y ocasionalmente uso de la fuerza": el poder está por todas partes; por tanto, el poder político no tiene nada distintivo o al revés todo es "político". Una de las más recientes, abanderada por uno de los coautores de un popular texto de psicología social (Gilovich, Keltner, \& Chen, 2018), es la que podemos denominar la "teoría de autoayuda del poder" (Keltner, 2016). Partiendo de algunas intuiciones correctas, como que el poder resulta de un "otorgamiento" (como nos decía Tarde), o que el poder genera narrativas de excepcionalísimo (como en otro lugar analizamos a propósito de la discusión del sufrimiento) se lanza a establecer toda una serie de afirmaciones bien cuestionables. La primera confusión viene de la afirmación que el poder se manifiesta en la influencia "positiva en el mundo" -lo cual no es más que un juicio de valor- porque requiere el reconocimiento de los demás, mezclando de este modo la creación -que requiere coaliciones no necesariamente construidas para hacer avanzar el bien en el mundo-y el ejercicio (que con frecuencia pasa por deshacer unas coaliciones y crear otras total o parcialmente diferentes). De esta confusión se deriva una segunda, según la cual las gentes llegan al poder por "los buenos motivos" y lo pierden "por los malos", es decir, llegan para ejercer una influencia positiva en el mundo y lo pierden cuando pasan a ejercer una influencia negativa, de modo que no hay nada distintivo en quien llega al poder, no hay hipótesis "psicopática". Y una tercera confusión que viene de asimilar los efectos psicológicos destructivos que la dominación induce en el dominado con los efectos de la ausencia de poder (ignorando, por otra parte, los múltiples mecanismos de auto - defensa, resistencia y creación de redes de solidaridad alternativos que están en la base de los movimientos de protesta).

\footnotetext{
3 “Poder significa la probabilidad de imponer la propia voluntad, dentro de una relación social, aun contra toda resistencia y cualquiera que sea el fundamento de esa probabilidad" (página43).
} 
4 "Entendemos aquí por "dominación" un estado de cosas por el cual una voluntad manifiesta ("mandato") del "dominador" o de los "dominadores" influye sobre los actos de otros (del "dominado" o de los "dominados"), de tal suerte que en un grado socialmente relevante estos actos tienen lugar como si los dominados hubieran adoptado por sí mismos y como máxima de su obrar el contenido del mandato (“obediencia" )"(pág. 699).

"Por otra parte, el enlace causal que liga el mandato a su cumplimiento puede adoptar formas muy diferentes. Desde el punto de vista puramente psicológico, un mandato puede ejercer su acción mediante “compenetración" -endopatía-, mediante "inspiración”, por "persuasión” racional o por combinación de algunas de estas tres formas capitales. Desde el punto de vista de su motivación concreta, un mandato puede ser cumplido por convencimiento de su rectitud, por sentimiento del deber, por temor, por "mera costumbre" o por conveniencia, sin que tal diferencia tenga necesariamente un significado sociológico" (pág. 699).

${ }^{5}$ Esto lleva inexorablemente a una versión esencialista del poder, en total contradicción con lo que hemos venido descubriendo sobre que es el poder y a confundir las condiciones de ejercicio del poder con su emergencia. Una gran parte del derecho constitucional, Kelsen incluido, basan su discurso en esta confusión y como consecuencia han permitido dotar a los Tribunales Constitucionales (y por extensión a los Tribunales de Justicia) de fundamentaciones ajenas a la soberanía del pueblo. Dicho en otros términos, las condiciones de un ejercicio desigual de toda relación de poder, se convierten en el fundamento de esa relación de poder: la obediencia se basa en la orden. Esta clase de disparate, solo deja abierto el camino a un tipo de movimiento de cambio político: el transgresor. No hay cambio evolutivo posible.

${ }^{6}$ Afirmación que no se opone, sino que es la cara de la moneda; la cruz es la "orden" que es lo que realmente (Durkheim, 1967, pág. 7) define como "hecho social" "Le fait social ne peut se définir par sa généralité à l'intérieur de la société. Caractères distinctifs du fait social : (i) son extériorité par rapport aux consciences individuelles; (ii) l'action coercitive qu'il exerce ou est susceptible d'exercer sur ces mêmes consciences ". Obviamente, quedarse solo en Durkheim obscurece el mundo, pero desde el punto de vista práctico es mucho más sencillo "hacer investigación" mirando solamente a esta parte del mundo. Y por lo mismo es mucho más probable "caer" en una definición esencialista del poder y una visión irenista de la política.

7 Como por otra parte la "teoría subjetiva del valor económico" ya sabe desde siempre; y el "mercado" es una dinámica de "creencias", como cualquiera que haya tratado de explicar el comportamiento de los mercados financieros, sea cual sea la sofisticación matemática que superponga, aprende (algunos todavía no). De hecho, los mercados pueden ser analizados como proyectos morales (Fourcade \& Healy, 2007) (Boltanski \& Thevenot, 2006).

${ }^{8}$ El efecto Halo (Rosenzweig, 2007) es el mismo que "explica" muchas de las disquisiciones sobre el "carisma" de los líderes, desde Max: Weber hasta hoy y la mayoría de las producciones de Think Thank: “мы так мало знаем, но кто-то всегда отвечает” (sabemos muy poco, pero alguien siempre responde).

${ }^{9} \mathrm{Y}$ no es un ejercicio exclusivamente retórico, como algunos "populistas" han pensado (a su costa). Se pueden inventariar los motivos (Burke, 1969a) y crear discursos alrededor de ellos (Burke, 1969) ; crear coaliciones de sujetos dispuestos a desafiar la orden y calificar a quién se esconde tras ella como parásito (con el destino implícito en esta descripción), ponerlas y mantenerlas en movimiento, es un trabajo de innovación titánico.

${ }^{10}$ El párrafo reza así: «Mais pourquoi sont-ils forcés parfois d'adhérer à un jugement qui flétrit leurs actes et contrarie leurs intérêts? Pourquoi leur arrive-t-il parfois de se réprouver eux-mêmes spontanément quand ils servent leur propre cause avec trop de zèle? Parce qu'on n'est pas toujours maître de croire ce qu'on désirerait croire, ce qu'on aurait intérêt à croire, parce que la croyance est, jusqu'à un certain point, autonome dans sa sphère et ne se laisse entamer qu'indirectement par le désir. Il y a des moments où un parti aurait intérêt à croire qu'il fait nuit en plein jour, mais où, si fort qu'il ferme les yeux, la lumière éclate $"$.

${ }^{11}$ Más discutible es su conclusión sobre la utilidad de la política; supuestamente esta contribuye a un fin civilizador superior: "Leur utilité est autre et plus élevée au point de vue de la civilisation générale. Elles 
ont pour effet, en abaissant les frontières des nations ou les murs de clôture des classes, de hâter, de favoriser l'élargissement graduel du champ social, et de préparer ainsi l'harmonie finale dans la lumière» (pág. 211).

12 (Benjamin, 1971). En el Capítulo: Para una crítica de la violencia del Angelus Novus (p171-199), analiza esa relación y nos dice que "La tarea de una crítica de la violencia puede definirse como la exposición de su relación con el derecho y con la justicia".

\section{Referencias citadas.}

Benjamin, W. (1971). Angelus Novus. Edhasa: Edhasa.

Blalock, H. (1985). Causal models in the social sciences. New York: Aldine Pub. Co.

Boétie. (1995). Discours de la servitude volontaire. Paris: Éd. Mille et une nuits.

Boltanski, L., \& Thevenot, L. (2006). On Justification: Economies of Worth. Princeton, NJ: Princeton Univ. Press.

Burke, K. (1969). A Rhetoric of Motives. University of California Press.

Burke, K. (1969a). A Grammar of Motives. Univeisiry of California Pres.

Cartwright, N. (2007). Hunting causes and using them : approaches in philosophy and economics. Cambridge New York: Cambridge University Press.

CIS. (2009). Ética pública y corrupción (Estudio n.o 2826). Centro de Investigaciones Sociológicas. Obtenido de http://www.cis.es/cis/opencm/ES/1_encuestas/estudios/ver.jsp?estudio=10684

Durkheim, E. (1967). Les règles dela méthode sociologique. UQAC.

Fourcade, M., \& Healy, K. (August de 2007). Moral Views of Market Society. Annual Review of Sociology, 33, 285-311. doi:10.1146/annurev.soc.33.040406.131642

Gilovich, T., Keltner, D., \& Chen, S. (2018). Social Psychology. W W Norton \& Co.

Greimas, A. J. (1987). Semantica Estructural. Gredos.

Greimas, A. J., \& Fontanille, J. (2002). Semiótica de las pasiones. Siglo XXI Editores.

Hayes, A. F. (2022). Introduction to Mediation, Moderation, and Conditional Process Analysis, Third Edition: A Regression-Based Approach. Guilford Pubn.

Hernan, M. A., \& Robins, J. M. (2023). Causal Inference. Taylor \& Francis Inc.

Jaccard, J., \& Jacoby, J. (2020). Theory Construction and Model-Building Skills, Second Edition: A Practical Guide for Social Scientists. GUILFORD PUBN.

Kant, I. (2011). La paz perpetua. Akal.

Keltner, D. (2016). The Power Paradox: How We Gain and Lose Influence. Penguin Publishing Group.

Lagasnerie, G. (2016). Juger. L'État pénal face à la Sociologie. Fayard.

Morgan, S. L., \& Winship, C. (January de 2015). Counterfactuals and Causal Inference.

Cambridge University Press. 
Nuñez, M. (2021). Corrupcion: ampliando el alcance. doi:10.31235/osf.io/6dm8q

Nuñez, M. (2022). Corrupción como estrategia de la anti-política. doi:10.31235/osf.io/5yubm

Pearl, J. (2000). Causality : models, reasoning, and inference. Cambridge, U.K. New York: Cambridge University Press.

Pearl, J., \& Mackenzie, D. (May de 2018). The Book of Why. Penguin Books Ltd.

Pearl, J., Glymour, M., \& Jewell, N. P. (2016). Causal Inference in Statistics. John Wiley and Sons Ltd.

Rosenzweig, P. (2007). The Halo Effect. Free Press.

Sloman, S., \& Fernbach, P. (2017). The Knowledge Illusion. Riverhead Books.

Tarde, G. (2003). Les transformations du pouvoir. Seuil.

Weber, M. (2002). Economia y Sociedad. FCE. 